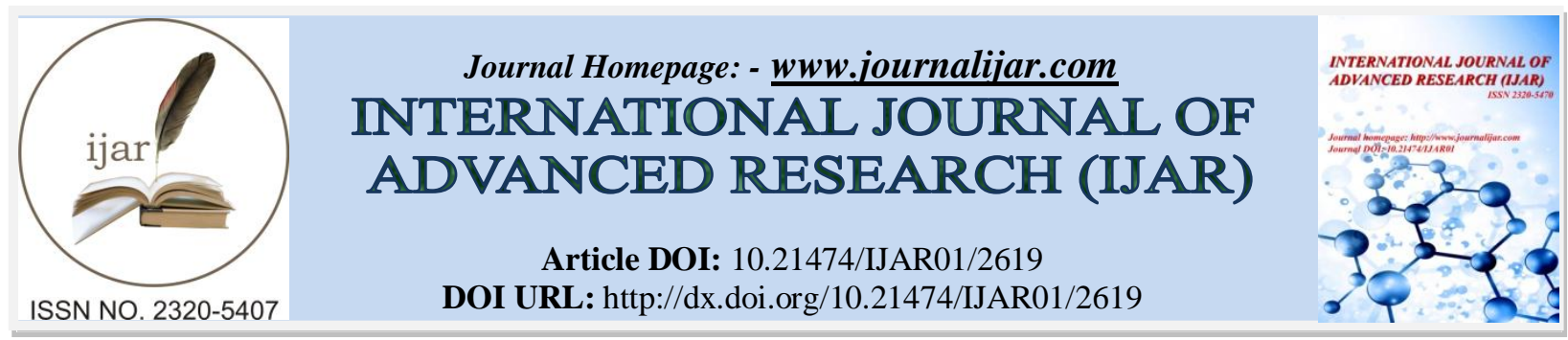

RESEARCH ARTICLE

\title{
EFFECT OF STORAGE CONDITIONS ON SEED QUALITY OF SOYBEAN AND IMPACT OF SEED PRODUCTION AREA.
}

\author{
Amol C. Vikhe. \\ Seed Testing Division, Department of Quality Assurance, Ajeet Seeds Pvt Ltd, Chitegaon, Aurangabad.
}

\section{Manuscript Info}

Manuscript History

Received: 30 October 2016

Final Accepted: 29 November 2016

Published: December 2016

Key words:-

Storage conditions, Temperature,

Relative humidity, seed Germination, mass weight, Shelf-life, Quality.

\section{Abstract}

The storage condition is most important factor in seed germination. The poor storage condition and relative environmental factors are affects on seed germination of soybean seeds. The deterioration of seed quality periodically goes on increasing during poor storage conditions. The poor storer seed shows significant affect on the electric conductivity, seed vigour and mass index. The subjected testing of the some lots of soybean cultivar MACS- 450 was grown in kharif season having different location of production center of Maharashtra state. The seed testing during initial, 3, 6, and 9 month in ambient and control conditions. The three lots were selected from each production center to test effective seed germinability and its Vigour at regular intervals, i.e. initial to 9 month, i.e. at validity period. The germination and Vigour significantly reduced in ambient condition from high to low percentage than that of control condition, which showed effective than the ambient one. It means that control storage condition is helpful for stable in vigor, seed germinability and maintaining shelf life of seed lots.

Copy Right, IJAR, 2016,. All rights reserved.

\section{Introduction:-}

The seeds of crops affected due to the many store grain pests such as, rice moth (Corcyra cephalonica staint), rice weevil (Sitophilus oryzae), pulse beetle (Bruchus spp.), kharpa beetle (Trogoderma granarium), Indian meal moth (Plodia interpunctella), and flat grain beetle.

The principal object of seed storage is to preserve storage temperature and moisture content on the vigor of economic crops from one season to another. Also prevent seed deterioration from environment and harmful organisms. Storage Welsh onion seeds. After two years storage, the seed temperature and moisture content are the most important quality declined as storage temperature and seed moisture factors affecting seed longevity, with seed moisture content increased. The effects of storage conditions.

Content usually being more influential than temperature on viability and vigor of onion seeds were studied. Many investigators reported that the speed of decline They reported that a complete pattern of loss in viability in seed quality is largely dependent on storage could be understood on the basis of seed moisture and temperature, relative humidity, seed moisture content, storage temperature and concluded that adoption of length of storage, type of seed and seed quality. The appropriate storage temperature and moisture control relation between seed deterioration and moisture technique would significantly affect onion seed quality. Thermodynamic properties of seeds were

Corresponding Author:- Amol C. Vikhe.

Address:- Seed Testing Division, Department of Quality Assurance, Ajeet Seeds Pvt Ltd, Chitegaon, 
examined. Khaldun and Haque studied the effect of temporal the thermodynamic properties lead to the loss of viability variation on the cucumber (Cucumis sativus) seed quality. And to an increase in seed leachate conductivity. Stum of They reported that the maximum germination parentage et al. tested 154 commercial seed lots of onion, stored was observed when moisture contents was 10.66 and For $1-10$ years. They concluded that as moisture content 10.95 but slightly decreased when the moisture content and storage period increases, the viability equation attained at 11.03 and 11.08 , respectively.

Changes occurring in seed during aging are significant as far as seed quality and longevity are concerned and are a consequence of the effects of different storage conditions. The obtained results showed that preservation of seed viability depended on storage condition and duration, as well as plant species. Accelerated aging test can be used to predict the length of storage life of sunflower and soybean seed. In comparison to sunflower seed, soybean seed is more sensitive to damage and reduced germination during storage. Balesevic-Tubic, S., Tatic et al ; (2010)

The influence of seed storage conditions and different temperatures $\left(5^{\circ} \mathrm{C}, 10^{\circ} \mathrm{C}, 15^{\circ} \mathrm{C}, 20^{\circ} \mathrm{C}, 25^{\circ} \mathrm{C}, 30^{\circ} \mathrm{C}\right.$ and $26^{\circ} \mathrm{C} / 21^{\circ} \mathrm{C}$ ) during germination and seedling development on seed germination, shoot length and germination rate of wild oat (Avena fatua L.) was examined. The results also showed that temperature had a significant effect on the percentage of germination and germination rate of A. fatua seeds. The highest total germination occurred at $15^{\circ} \mathrm{C}$ temperature. Marija Saric-Krsmanovic, Jelena Gajic Umiljendic et.al ;(2015).

Seeds of four vegetable crops; carrot (Daucus carota L. cv Nantes 2-Tito), cucumber (Cucumis sativus L. cv Special), onion (Alium cepa L. cv Red Creole ) and tomato (Lycopersicon esculentum Mill. Cv Tanshet Star) were stored under a wide range of temperature $\left(5,15,25\right.$ and $\left.35^{\circ} \mathrm{C}\right)$ and relative humidity $(\mathrm{RH})(11.3,22.5,32.5,43.2$, 58.4, 75.3 and 84.3\%) conditions for various storage periods (1,3, 6, 9 and 12 months), seeds stored at $35^{\circ} \mathrm{C}$ had the lowest SGP and GCV and the longest MGT. RH up to 58.4\% had no significant effect on SGP while higher levels of RH significantly lowered SGP and MGT. The highest RH levels (75 and 84\%) showed an obvious decrease in seed quality by lowering SGP and increasing MGT. Abdullah M. Alhamdan, Abdullah A. Alsadon et.al ;( 2011).

The objective of the study was to determine seed quality response of twelve soybean lots stored under a wide range of temperature and relative humidity conditions for various periods.Quality parameters used in this study include seed germination percentage, seed index mass, vigour index length.

\section{Materials And Methods:- \\ Materials:}

Seed material of Soybean MACS-450 variety which is growing in different production centers viz; Sillod, Gangapur, D.Raja, and Newasa during kharif 2014 were used for the study. The climatic and environmental conditions are different. The storage conditions affect on the seed quality parameters such as Germination, seed vigour index (Length), seed vigour index (mass) during regular interval of time. Three lots of said variety taken for the testing from each production center; the lots were stored in ambient $\left(29^{\circ} \mathrm{c} \& 75 \% \mathrm{RH}\right)$ and control $\left(16^{\circ} \mathrm{C} \& 30 \% \mathrm{RH}\right)$ condition. They were tested from initial to nine months in both the conditions. The following parameters were tested according to ISTA standards.

1. Germination

2. Seedling Mass Weight

3. Seedling Length

4. Seedling Vigour Index Length

5. Seedling Vigour Index Mass

Germination:-

Germination is a process in which the emergence of plumule and radicle in presence of favourable conditions such as light, temp., water, humidity.

Equipments:-

1. Electric Oven with capacity $250^{\circ} \mathrm{c}$.

2. Germination walking Chamber with aseptic condition.

3. Evaluation Board/Counting board.

4. Petri plates, Pencil, Eraser, Thread, Scale, Record Book.

5. Soybean Cultivar Samples

6. Analytical Balance

7. Germination Media (Paper) 


\section{Methods:}

1. The sample is prepared for testing with their labels.

2. Spread a paper towel on a flat surface and moisten with water until it is thoroughly damp.

3. Place a total of 20 seeds in two Replications i.e.10 seeds in each replication in rows on the towel.

4. Moisten a second towel and carefully place onto the first paper towel.

5. Roll up the two towels with the seeds in-between and place in a sealed container that will retain the moisture. Place the container in an area of relatively stable temperature $\left(22-25^{\circ} \mathrm{C}\right) \& \mathrm{RH}(65-70 \%)$ unless otherwise instructed. Avoid areas where direct sunlight with its heating effect strikes the container.

6. Mark the container with the date and variety Lots of seed.

7. After the required germination period ( 5 days), remove the towels from the container.

8. Count the seedlings that have shoots longer than $1 \frac{1}{2}$ inches (and at least one strong root) as viable seeds in the germination rate. Seedlings exhibiting short shoots and/or roots less than $1 \frac{1}{2}$ inches would probably not germinate soon enough in our cool soils to contribute significantly to the yield.

9. Determine the actual percent of germination. In this case it is the number of seeds exhibiting strong germination.

10. The seedlings categorized into Normal seedling, abnormal seedlings, Fresh ungerminated seeds, hard seeds, Dead seeds.

Formula-Total Number of Germinated Seeds /Total Number of Seeds Tested $\times 100$.

Normal Seedlings: The seedlings shows well developed plumule and radicle and further developed into normal plant.

Abnormal Seedlings: The discontinued growth of plumule and radicle or the absence of any one of the essential structure.

Fresh Ungerminated Seeds: The seed absorbs the moisture after end of test period but not shows any structure and remains as it is.

Hard Seeds: At the end of test period seed doesn't absorbs moisture and remains as it is., when pressing it looks hard.

Dead Seeds: The decaying matter emerges out from the seed when pressing the seed after test period is dead seeds.

2. Seedling mass weight

The fresh weight of Root and Shoot taken separately in Gms and kept in Petridishes. The fresh weight taken in analytical weighing machine. The lot showing maximum weight is considered as more vigorous.

3. Seedling Length

Each and every seedling (Root and Shoot) were measured separately in cms. The lot showing maximum seedling length is considered as vigorous.

They were kept for Oven drying for 18 hours at $103^{\circ} \mathrm{c}$ temperature.

4. Seed Vigour Index Length

The total length of Root and Shoot measured and then mean of total length taken for calculating the seed vigour index length. The vigour index length calculated by following formula. A combination of standard germination test with seedling length provides board evaluation of seedling vigour. Seed lot with high vigour index is considered as vigorous (Abdul Baki Anderson, 1973).

Vigour Length Index \%= Germination $\times$ Total seedling length on the day of final count.

5. Seed Vigour Index Mass

After oven drying for 18 hours at $103^{\circ} \mathrm{c}$ temperature the dry matter kept for cooling and weight taken after cooling. seed vigour index mass calculated by following formula.

Vigour Mass Index \%= Germination Dry weight of seedling after the final count.

\section{Results And Discussion:-}

Table-1:- Germination Soybean MACS-450

\begin{tabular}{|c|c|c|c|c|c|c|c|c|c|c|c|c|}
\hline \multicolumn{13}{|c|}{ Comparative Testing Soybean MACS-450 } \\
\hline \multirow{3}{*}{$\begin{array}{l}\text { Testing } \\
\text { Period }\end{array}$} & \multicolumn{12}{|c|}{ Germination \% } \\
\hline & \multicolumn{12}{|c|}{ Lot. No. } \\
\hline & 8205 & 8204 & 8222 & 8067 & 8066 & 8063 & 8256 & 8253 & 8252 & 8221 & 8220 & 8173 \\
\hline $\begin{array}{l}\text { Initial } \\
\text { A }\end{array}$ & 68.00 & 84.00 & 83.00 & 80.00 & 70.00 & 92.00 & 80.00 & 91.00 & 91.00 & 85.00 & 80.00 & 89.00 \\
\hline $\begin{array}{l}3 \\
\text { Month } \\
\text { A }\end{array}$ & 60.00 & 76.00 & 80.00 & 70.00 & 68.00 & 85.00 & 75.00 & 82.00 & 80.00 & 79.00 & 69.00 & 76.00 \\
\hline
\end{tabular}




\begin{tabular}{|c|c|c|c|c|c|c|c|c|c|c|c|c|}
\hline $\begin{array}{l}6 \\
\text { Month } \\
\text { A }\end{array}$ & 54.00 & 73.00 & 74.00 & 53.00 & 67.00 & 81.00 & 50.00 & 78.00 & 75.00 & 76.00 & 46.00 & 70.00 \\
\hline $\begin{array}{l}9 \\
\text { Month } \\
\text { A }\end{array}$ & 50.00 & 63.00 & 72.00 & 73.00 & 64.00 & 83.00 & 71.00 & 73.00 & 84.00 & 78.00 & 70.00 & 68.00 \\
\hline $\begin{array}{l}\text { Initial } \\
\mathrm{C}\end{array}$ & 68.00 & 84.00 & 83.00 & 80.00 & 70.00 & 92.00 & 80.00 & 91.00 & 91.00 & 85.00 & 80.00 & 89.00 \\
\hline $\begin{array}{l}3 \\
\text { Month } \\
\text { C }\end{array}$ & 65.00 & 80.00 & 85.00 & 80.00 & 72.00 & 90.00 & 75.00 & 82.00 & 84.00 & 80.00 & 75.00 & 75.00 \\
\hline $\begin{array}{l}6 \\
\text { Month } \\
\text { C }\end{array}$ & 62.00 & 78.00 & 88.00 & 80.00 & 75.00 & 87.00 & 69.00 & 85.00 & 87.00 & 77.00 & 71.00 & 63.00 \\
\hline $\begin{array}{l}9 \\
\text { Month } \\
\text { C } \\
\end{array}$ & 75.00 & 75.00 & 85.00 & 83.00 & 70.00 & 86.00 & 70.00 & 86.00 & 87.00 & 85.00 & 79.00 & 73.00 \\
\hline $\mathrm{SD} \pm$ & $\begin{array}{l} \pm 8.08 \\
4 \\
\end{array}$ & $\begin{array}{l} \pm 6.80 \\
2 \\
\end{array}$ & $\begin{array}{l} \pm 5.59 \\
9 \\
\end{array}$ & $\begin{array}{l} \pm 9.83 \\
3 \\
\end{array}$ & $\begin{array}{l}3.29 \\
5 \\
\end{array}$ & $\begin{array}{l} \pm 4.07 \\
0 \\
\end{array}$ & $\begin{array}{l} \pm 9.55 \\
8\end{array}$ & $\begin{array}{l} \pm 6.16 \\
4\end{array}$ & $\begin{array}{l} \pm 5.43 \\
6\end{array}$ & $\begin{array}{l} \pm 3.81 \\
4\end{array}$ & $\begin{array}{l} \pm 11.15 \\
7\end{array}$ & $\begin{array}{l} \pm 9.36 \\
4\end{array}$ \\
\hline $\mathrm{SE} \pm$ & $\begin{array}{l} \pm 2.85 \\
8\end{array}$ & $\begin{array}{l} \pm 2.40 \\
5\end{array}$ & $\begin{array}{l} \pm 1.98 \\
0\end{array}$ & $\begin{array}{l} \pm 3.47 \\
7\end{array}$ & $\begin{array}{l} \pm 1.16 \\
5\end{array}$ & $\begin{array}{l} \pm 1.43 \\
9\end{array}$ & $\begin{array}{l} \pm 3.37 \\
9\end{array}$ & $\begin{array}{l} \pm 2.17 \\
9\end{array}$ & $\begin{array}{l} \pm 1.92 \\
2\end{array}$ & $\begin{array}{l} \pm 1.34 \\
8\end{array}$ & \pm 3.945 & $\begin{array}{l} \pm 3.31 \\
1\end{array}$ \\
\hline
\end{tabular}

Table-2:- Mass Index Soybean MACS-450

\begin{tabular}{|c|c|c|c|c|c|c|c|c|c|c|c|c|}
\hline \multicolumn{13}{|c|}{ Comparative Testing Soybean MACS-450 } \\
\hline \multirow{3}{*}{$\begin{array}{l}\text { Testin } \\
\mathrm{g} \\
\text { Period }\end{array}$} & \multicolumn{12}{|c|}{ Mass. Index \% } \\
\hline & \multicolumn{12}{|c|}{ Lot. No. } \\
\hline & 8205 & 8204 & 8222 & 8067 & 8066 & 8063 & 8256 & 8253 & 8252 & 8221 & 8220 & 8173 \\
\hline $\begin{array}{l}\text { Initial } \\
\text { A }\end{array}$ & 12.24 & 18.90 & 14.94 & 16.40 & 16.10 & 23.92 & 17.20 & 23.66 & 23.21 & 16.58 & 21.60 & 22.25 \\
\hline $\begin{array}{l}3 \\
\text { Month } \\
\text { A }\end{array}$ & 10.12 & 12.17 & 18.20 & 14.92 & 18.02 & 26.12 & 10.12 & 20.18 & 23.12 & 18.14 & 12.16 & 12.11 \\
\hline $\begin{array}{l}6 \\
\text { Month } \\
\text { A } \\
\end{array}$ & 9.24 & 13.64 & 17.94 & 9.12 & 18.95 & 24.59 & 9.66 & 20.08 & 22.59 & 16.04 & 9.70 & 10.75 \\
\hline $\begin{array}{l}9 \\
\text { Month } \\
\text { A }\end{array}$ & 6.55 & 9.64 & 11.76 & 8.23 & 12.63 & 22.43 & 10.31 & 15.10 & 21.59 & 17.25 & 16.02 & 13.38 \\
\hline $\begin{array}{l}\text { Initial } \\
\mathrm{C}\end{array}$ & 12.24 & 18.90 & 14.94 & 16.40 & 16.10 & 23.92 & 17.20 & 23.66 & 23.21 & 16.58 & 21.60 & 22.25 \\
\hline $\begin{array}{l}3 \\
\text { Month } \\
\mathrm{C}\end{array}$ & 12.15 & 16.15 & 16.18 & 17.12 & 18.17 & 27.24 & 13.10 & 21.16 & 23.17 & 21.13 & 18.15 & 18.02 \\
\hline $\begin{array}{l}6 \\
\text { Month } \\
\text { C }\end{array}$ & 14.32 & 14.99 & 21.43 & 18.17 & 19.55 & 29.52 & 15.34 & 20.97 & 22.65 & 20.35 & 16.20 & 16.46 \\
\hline $\begin{array}{l}9 \\
\text { Month } \\
\text { C } \\
\end{array}$ & 12.46 & 13.23 & 20.64 & 16.70 & 16.12 & 23.07 & 12.25 & 19.32 & 23.67 & 21.13 & 16.40 & 16.60 \\
\hline $\mathrm{SD} \pm$ & $\begin{array}{l} \pm 2.42 \\
0\end{array}$ & $\begin{array}{l} \pm 3.22 \\
4\end{array}$ & $\begin{array}{l} \pm 3.20 \\
1\end{array}$ & $\begin{array}{l} \pm 3.79 \\
3\end{array}$ & $\begin{array}{l} \pm 2.21 \\
0\end{array}$ & $\begin{array}{l} \pm 2.37 \\
4\end{array}$ & $\begin{array}{l} \pm 3.11 \\
2\end{array}$ & $\begin{array}{l} \pm 2.71 \\
1\end{array}$ & $\begin{array}{l} \pm 0.62 \\
9\end{array}$ & $\begin{array}{l} \pm 2.14 \\
6\end{array}$ & $\begin{array}{l} \pm 4.14 \\
3\end{array}$ & $\begin{array}{l} \pm 4.31 \\
7\end{array}$ \\
\hline $\mathrm{SE} \pm$ & $\begin{array}{l} \pm 0.85 \\
5\end{array}$ & $\begin{array}{l} \pm 1.14 \\
0\end{array}$ & $\begin{array}{l} \pm 1.13 \\
2\end{array}$ & $\begin{array}{l} \pm 1.34 \\
1\end{array}$ & $\begin{array}{l} \pm 0.78 \\
1\end{array}$ & $\begin{array}{l} \pm 0.83 \\
9\end{array}$ & $\begin{array}{l} \pm 1.10 \\
0\end{array}$ & $\begin{array}{l} \pm 0.95 \\
8\end{array}$ & $\begin{array}{l} \pm 0.22 \\
2\end{array}$ & $\begin{array}{l} \pm 0.75 \\
9\end{array}$ & $\begin{array}{l} \pm 1.46 \\
5\end{array}$ & $\begin{array}{l} \pm 1.52 \\
6\end{array}$ \\
\hline
\end{tabular}


Table-3:- Vigour Index Length Soybean MACS-450

\begin{tabular}{|c|c|c|c|c|c|c|c|c|c|c|c|c|}
\hline \multicolumn{13}{|c|}{\begin{tabular}{|l} 
Comparative Testing Soybean MACS-450 \\
\end{tabular}} \\
\hline \multirow{3}{*}{$\begin{array}{l}\text { Testi } \\
\text { ng } \\
\text { Perio } \\
\text { d }\end{array}$} & \multicolumn{12}{|c|}{ Vigour Index Length \% } \\
\hline & \multicolumn{12}{|c|}{ Lot. No. } \\
\hline & 8205 & 8204 & 8222 & 8067 & 8066 & 8063 & 8256 & 8253 & 8252 & 8221 & 8220 & 8173 \\
\hline $\begin{array}{l}\text { Initia } \\
1 \mathrm{~A}\end{array}$ & $\begin{array}{l}947.5 \\
8\end{array}$ & $\begin{array}{l}1582 . \\
56\end{array}$ & $\begin{array}{l}965.7 \\
1\end{array}$ & $\begin{array}{l}1344 . \\
80\end{array}$ & $\begin{array}{l}1100 . \\
75\end{array}$ & $\begin{array}{l}1747 . \\
54\end{array}$ & $\begin{array}{l}1254 . \\
80\end{array}$ & $\begin{array}{l}1664 . \\
85\end{array}$ & $\begin{array}{l}1557 . \\
47\end{array}$ & $\begin{array}{l}1330 . \\
25\end{array}$ & $\begin{array}{l}1459 . \\
60\end{array}$ & $\begin{array}{l}1627 . \\
37\end{array}$ \\
\hline \begin{tabular}{|l|}
3 \\
Mont \\
h A
\end{tabular} & $\begin{array}{l}770.1 \\
2\end{array}$ & $\begin{array}{l}1090 . \\
12\end{array}$ & $\begin{array}{l}1014 . \\
16\end{array}$ & $\begin{array}{l}812.0 \\
2\end{array}$ & $\begin{array}{l}914.1 \\
0\end{array}$ & $\begin{array}{l}1615 . \\
12\end{array}$ & $\begin{array}{l}968.0 \\
6\end{array}$ & $\begin{array}{l}1217 . \\
90\end{array}$ & $\begin{array}{l}1216 . \\
91\end{array}$ & $\begin{array}{l}1141 . \\
60\end{array}$ & $\begin{array}{l}920.1 \\
4\end{array}$ & $\begin{array}{l}818.0 \\
0\end{array}$ \\
\hline $\begin{array}{l}6 \\
\text { Mont } \\
\text { h A }\end{array}$ & $\begin{array}{l}647.8 \\
5\end{array}$ & $\begin{array}{l}1080 . \\
04\end{array}$ & $\begin{array}{l}1188 . \\
81\end{array}$ & $\begin{array}{l}582.7 \\
4\end{array}$ & $\begin{array}{l}1044 . \\
53\end{array}$ & $\begin{array}{l}1560 . \\
47\end{array}$ & $\begin{array}{l}697.0 \\
0\end{array}$ & $\begin{array}{l}1369 . \\
68\end{array}$ & $\begin{array}{l}1345 . \\
50\end{array}$ & $\begin{array}{l}1031 . \\
70\end{array}$ & $\begin{array}{l}570.8 \\
6\end{array}$ & $\begin{array}{l}747.9 \\
5\end{array}$ \\
\hline $\begin{array}{l}9 \\
\text { Mont } \\
\text { h A }\end{array}$ & $\begin{array}{l}408.5 \\
0\end{array}$ & $\begin{array}{l}710.9 \\
6\end{array}$ & $\begin{array}{l}763.2 \\
0\end{array}$ & $\begin{array}{l}684.7 \\
4\end{array}$ & $\begin{array}{l}790.7 \\
2\end{array}$ & $\begin{array}{l}1448 . \\
77\end{array}$ & $\begin{array}{l}757.2 \\
2\end{array}$ & $\begin{array}{l}969.0 \\
8\end{array}$ & $\begin{array}{l}1403 . \\
64\end{array}$ & $\begin{array}{l}1134 . \\
12\end{array}$ & $\begin{array}{l}1019 . \\
90\end{array}$ & $\begin{array}{l}677.2 \\
8\end{array}$ \\
\hline $\begin{array}{l}\text { Initia } \\
1 \mathrm{C}\end{array}$ & $\begin{array}{l}947.5 \\
8\end{array}$ & $\begin{array}{l}1582 . \\
56\end{array}$ & $\begin{array}{l}965.7 \\
1\end{array}$ & $\begin{array}{l}1344 . \\
80\end{array}$ & $\begin{array}{l}1100 . \\
75\end{array}$ & $\begin{array}{l}1747 . \\
54\end{array}$ & $\begin{array}{l}1254 . \\
80\end{array}$ & $\begin{array}{l}1664 . \\
85\end{array}$ & $\begin{array}{l}1557 . \\
47\end{array}$ & $\begin{array}{l}1330 . \\
25\end{array}$ & $\begin{array}{l}1459 . \\
60\end{array}$ & $\begin{array}{l}1627 . \\
37\end{array}$ \\
\hline $\begin{array}{l}3 \\
\text { Mont } \\
\text { h C }\end{array}$ & $\begin{array}{l}892.1 \\
4\end{array}$ & $\begin{array}{l}1095 . \\
16\end{array}$ & $\begin{array}{l}1218 . \\
20\end{array}$ & $\begin{array}{l}1118 . \\
26\end{array}$ & $\begin{array}{l}1020 . \\
17\end{array}$ & $\begin{array}{l}1920 . \\
00\end{array}$ & $\begin{array}{l}1012 . \\
08\end{array}$ & $\begin{array}{l}1290 . \\
16\end{array}$ & $\begin{array}{l}1318 . \\
20\end{array}$ & $\begin{array}{l}1281 . \\
18\end{array}$ & $\begin{array}{l}1080 . \\
89\end{array}$ & $\begin{array}{l}1008 . \\
40\end{array}$ \\
\hline $\begin{array}{l}6 \\
\text { Mont } \\
\text { h C }\end{array}$ & $\begin{array}{l}902.4 \\
1\end{array}$ & $\begin{array}{l}1027 . \\
65\end{array}$ & $\begin{array}{l}1383 . \\
36\end{array}$ & $\begin{array}{l}1130 . \\
40\end{array}$ & $\begin{array}{l}1189 . \\
88\end{array}$ & $\begin{array}{l}2003 . \\
61\end{array}$ & $\begin{array}{l}1108 . \\
49\end{array}$ & $\begin{array}{l}1274 . \\
58\end{array}$ & $\begin{array}{l}1474 . \\
65\end{array}$ & $\begin{array}{l}1366 . \\
75\end{array}$ & $\begin{array}{l}1028 . \\
71\end{array}$ & $\begin{array}{l}999.1 \\
8\end{array}$ \\
\hline $\begin{array}{l}9 \\
\text { Mont } \\
\text { h C }\end{array}$ & $\begin{array}{l}857.2 \\
5\end{array}$ & $\begin{array}{l}1015 . \\
88\end{array}$ & $\begin{array}{l}1339 . \\
60\end{array}$ & $\begin{array}{l}1234 . \\
63\end{array}$ & $\begin{array}{l}1081 . \\
50\end{array}$ & $\begin{array}{l}1639 . \\
59\end{array}$ & $\begin{array}{l}984.5 \\
5\end{array}$ & $\begin{array}{l}1319 . \\
24\end{array}$ & $\begin{array}{l}1580 . \\
36\end{array}$ & $\begin{array}{l}1337 . \\
05\end{array}$ & $\begin{array}{l}1045 . \\
17\end{array}$ & $\begin{array}{l}1059 . \\
60\end{array}$ \\
\hline $\mathrm{SD} \pm$ & $\begin{array}{l} \pm 186 \\
333\end{array}$ & $\begin{array}{l} \pm 295 \\
537\end{array}$ & $\begin{array}{l} \pm 212 . \\
549\end{array}$ & $\begin{array}{l} \pm 298 \\
732\end{array}$ & $\begin{array}{l} \pm 124 \text {. } \\
910\end{array}$ & $\begin{array}{l} \pm 184 \text {. } \\
270\end{array}$ & $\begin{array}{l} \pm 204 \\
800\end{array}$ & $\begin{array}{l} \pm 230 . \\
312\end{array}$ & $\begin{array}{l} \pm 132 \text {. } \\
456\end{array}$ & $\begin{array}{l} \pm 123 . \\
991\end{array}$ & $\begin{array}{l} \pm 287 \\
861\end{array}$ & $\begin{array}{l} \pm 368 . \\
721\end{array}$ \\
\hline $\mathrm{SE} \pm$ & $\begin{array}{l} \pm 65.8 \\
88\end{array}$ & $\begin{array}{l} \pm 104 . \\
503\end{array}$ & $\begin{array}{l} \pm 75.1 \\
59\end{array}$ & $\begin{array}{l} \pm 105 \\
633\end{array}$ & $\begin{array}{l} \pm 44.1 \\
69\end{array}$ & $\begin{array}{l} \pm 65.1 \\
59\end{array}$ & $\begin{array}{l} \pm 72.4 \\
18\end{array}$ & $\begin{array}{l} \pm 81.4 \\
40\end{array}$ & $\begin{array}{l} \pm 46.8 \\
37\end{array}$ & $\begin{array}{l} \pm 43.8 \\
44\end{array}$ & $\begin{array}{l} \pm 101 \text {. } \\
789\end{array}$ & $\begin{array}{l} \pm 130 . \\
382\end{array}$ \\
\hline
\end{tabular}

Graph-1:- Germination Soybean MACS-450
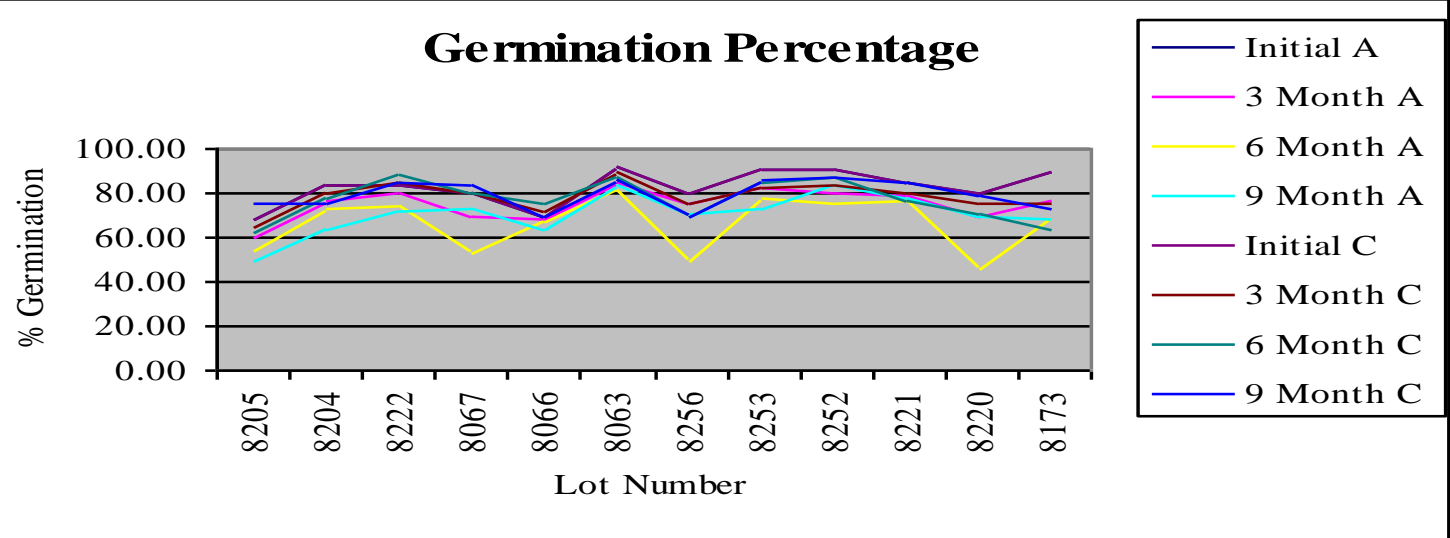
Graph-2:- Mass Index Soybean MACS-450

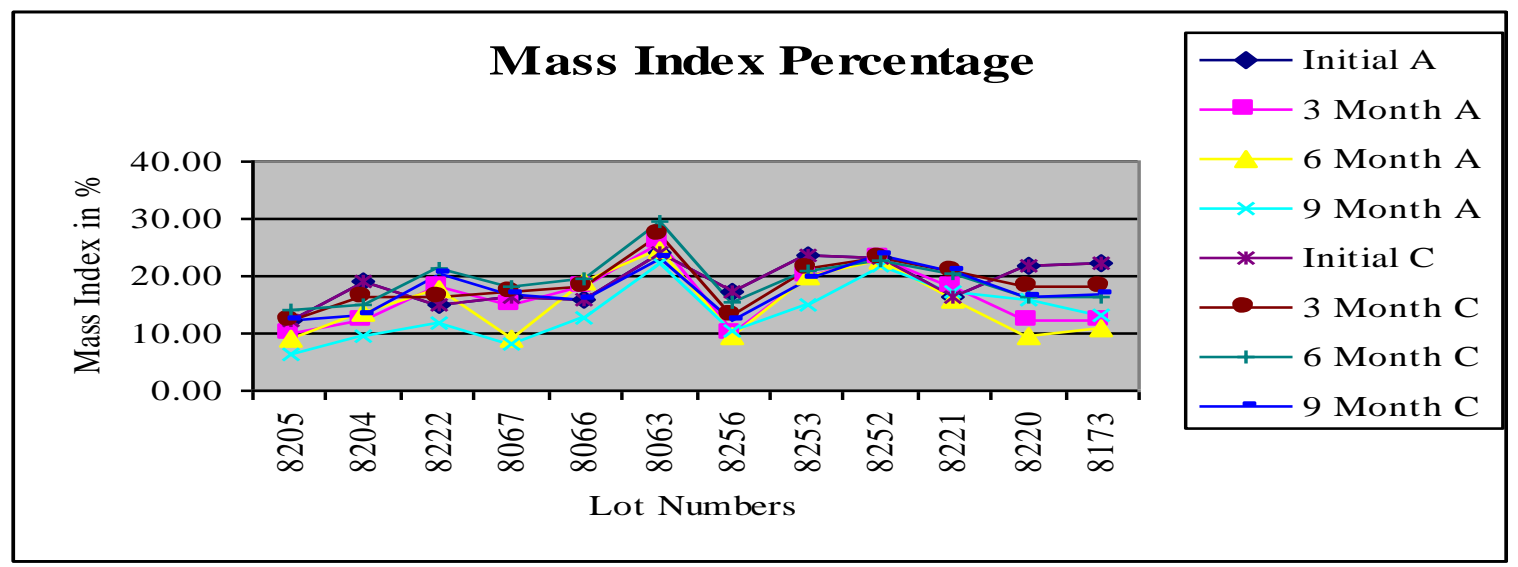

Graph-3:- Vigour Index Length Soybean MACS-450

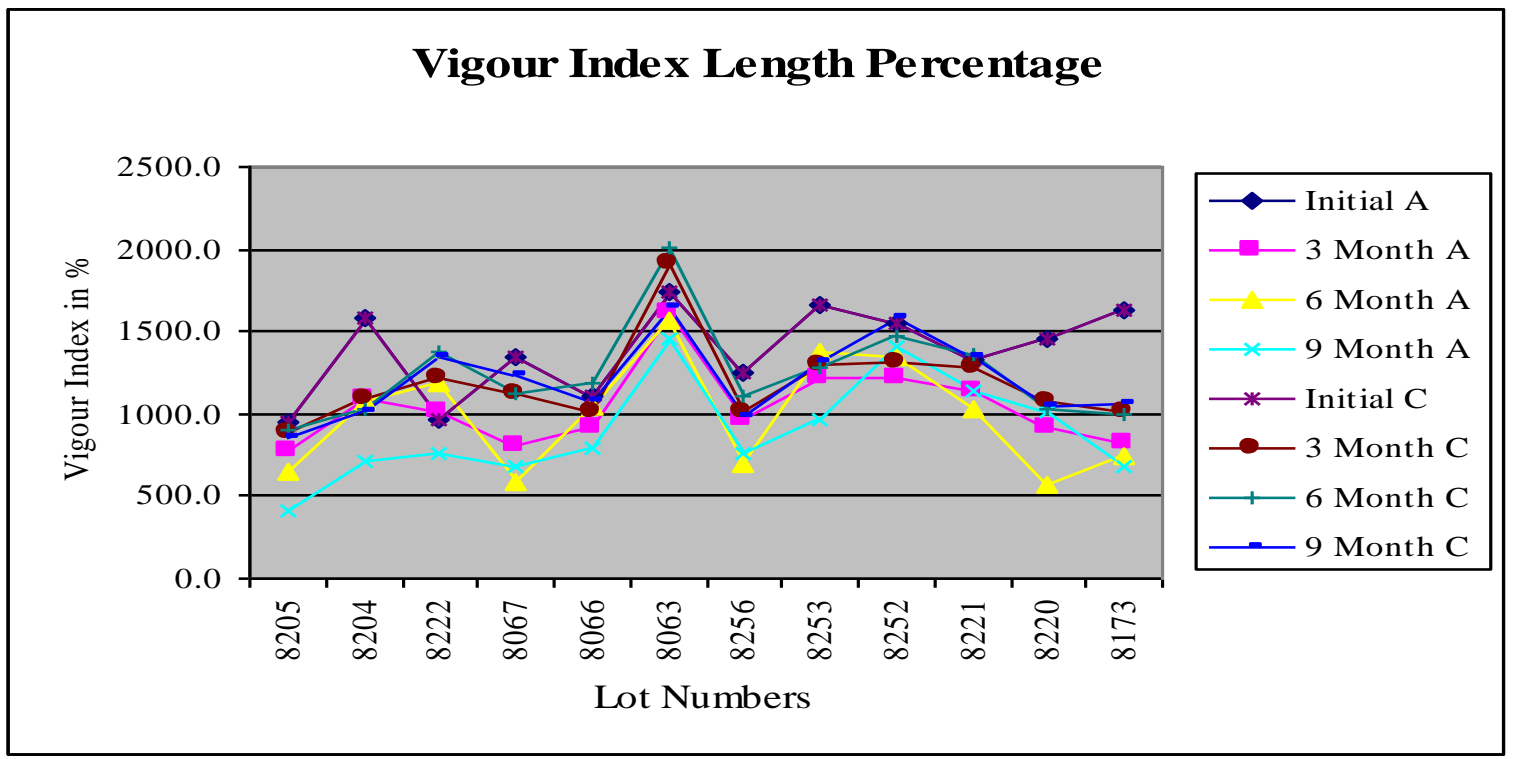

\section{Discussion:-}

All above experimental results showed the significant correlation in storage conditions i.e. ambient and control condition. Several techniques which are used for seed preservation to maintain or to stabilize the viability of seed (to maintain seed quality) by researchers. The 12 samples of soybean tested for this point of view to test the seed quality after initial, 3, 6,9months intervals during storage conditions in ambient $\left(29^{\circ} \mathrm{c} \& 75 \% \mathrm{RH}\right)$ and control $\left(16^{\circ} \mathrm{C} \& 30 \% \mathrm{RH}\right)$ condition; and we get the appropriate results regarding this.

The seed quality is stabilize for sixth month, later on it slows down after specific time duration period or it is deteriorates in some periods.

The testing of these for seed germination, seedling mass wt., vigor index length, vigor index mass. The twelve samples of variety MACS- 450 i.e.

$8205,8204,8222,8067,8066,8063,8256,8253,8252,8221,8220,8173 ; \quad$ Showed germination $68,60,54,50 ; 84,76,73,63 ; 83,80,74,72 ; 80,70,53,73 ; 70,68,67,64 ; 92,85,81,83 ; 80,75,50,71 ; 91,82,78,73 ; 91,80,75,84 ; 85$, $79,76,78 ; 80,69,46,70 ; 89,76,70,68 ;$ respectively,after initial,3,6,9monthinambientcondition.

The control condition showed germination,

$65,62,75 ; 80,78,75 ; 85,88,85 ; 80,80,83 ; 72,75,70 ; 90,87,86 ; 75,69,70 ; 82,85,86 ; 84,87,87 ; 80,77,85 ; 75,71,79 ; 75,63,73 ;$ in $3,6,9$ month respectively. The germination percentage of samples is increased or stable in control condition except sample number 7and12; which showed lowest figures; than that of ambient condition. In relationship with mass 
index, it gradually decreased after testing periods of initial, 3, 6, 9months, in ambient and control condition. Mass indexes as follow,

$12.24,10.1240,9.2390,6.5450 ; 18.90,12.1660,13.6350,9.6390 ; 14.94,18.2042,17.9350,11.7612 ; 16.40,14.9212,9.1195$, $8.2271 ; 16.10,18.0245,18.9545,12.6336 ; 23.92,26.1215,24.5850,22.4266 ; 17.20,10.1217,9.6625,10.3057 ; 23.66,20.18$ $20,20.0780,15.1001 ; 23.21,23.1214,22.5925,21.5922 ; 16.58,18.1414,16.0350,17.2536 ; 21.60,12.1611,9.6985,16.0195$ ;22.25,12.1117,10.7510,13.3790;

In ambient condition from initial to 9 month samples respectively. Incontrolcondition $12.1520,14.3150,12.4575 ; 16.1522,14.99,13.23 ; 16.1812,21.4250,20.6380 ; 17.1224,18.1700,16.703$ $8 ; 18.1712,19.5500,16.1175 ; 27.2418,29.5150,23.0652 ; 13.1020,15.3350,12.25 ; 21.1621,20.9700,19.3199 ; 23.1720,22$. $6500,23.6684 ; 21.1290,20.3450,21.1268 ; 18.1517,16.20,16.3965 ; 18.0222,16.4550,16.6002 ;$ respectively in $3,6,9$ month.

The Vigor index length also declined during storage periods. In ambient it is $947.58,770.12,647.85,408.50 ; 1582.56,1090.12,1080.04,710.96 ; 965.71,1014.1622,1188.81,763.20 ; 1344.80,812.02,5$ 82.74,684.74; 1100.75,914.10,1044.53,790.92;1747.54,1615.12,1560.47,1448.77;1254.80,968.06,697.00,757.22;166 4.85,1217.90,1369.68,969.08;1557.47,1216.91,1345.50,1403.64;1330.25,1141.60,1031.70,1134.12;1459.60,920.14, $570.86,1019.90 ; 1627.37,818.00,747.95,677.28$;respectively.

In initial, 3, 6,9month.In cold storage;

$892.14,902.41,857.25 ; 1095.16,1027.65,1015.88 ; 1218.20,1383.36,1339.60 ; 1118.26,1130.40,1234.63 ; 1020.17,1189$. $88,1081.50 ; 1920.00,2003.61,1639.59 ; 1012.08,1108.49,984.55 ; 1290.16,1274.58,1319.24 ; 1318.20,1474.64,1580.36$; $1281.18,1366.75,1337.05 ; 1080.89,1028.71,1045.17 ; 1008.40,999.18,1059.60$; respectively in 3,6,9month.

\section{Conclusion:-}

In present research work revealed that the optimum germination percentage level maintained at specific time of period; but the parameters regarding vigor index mass, length are declined during the storage in regular intervals (3, 6,9 month) in both the conditions (Ambient and control). The level of declination in cold (control) storage condition $\left(16^{\circ} \mathrm{c} \& 30 \% \mathrm{RH}\right)$ is less than the ambient condition $\left(29^{\circ} \mathrm{c} \& 75 \% \mathrm{RH}\right)$. Hence the stored samples in control condition stable the viability; so advise to followers to store samples in control conditions.

\section{References:-}

1. Balesevic-Tubic, S., Tatic et al ; (2010) SEED VIABILITY OF OIL CROPS DEPENDING ON STORAGE CONDITIONS. HELIA, 33, Nr. 52, p.p. 153-160, (2010).

2. Marija Saric-Krsmanovic, Jelena Gajic Umiljendic et.al ;( 2015) Impact of storage conditions on seed germination and seedling growth of wild oat (Avena fatua L.) at different temperatures. Pestic. Phytomed. (Belgrade), 30(4), 2015, 243-248. DOI: 10.2298/PIF1504243S.

3. Abdullah M. Alhamdan, Abdullah A. Alsadon et.al;(2011). Influence of Storage Conditions on Seed Quality and Longevity of Four Vegetable Crops. American-Eurasian J. Agric. \& Environ. Sci., 11 (3): 353-359, 2011ISSN 1818-6769@ IDOSI Publications, 2011.

4. Seed Technology (2000) - Dhirendra Khare \& Mohan Bhale, Scientific Publishers, Jodhpur.

5. Seed Technology- Ratanlal Agrawal, Oxford \& IBH Publishing CO. PVT. LTD. New Delhi

6. Soybean Seed and Seedling Diseases. Crop Focus, Dupont pioneer agronomy science.

7. ISTA, 1999. International Rules for Seed Testing. International Seed Testing Association,

8. Switzerland.

9. Christensen, C.M., 1971. Evaluating conditions and storability of sunflower seeds. J. Stored Prod. Res. 7: 163169.

10. Balesevic-Tubic, S., Tatić, M., Hrustić, M., Miladinović, J., Maksimović, L., 2007b. The influence of aging process on germination and seedling growth of sunflower seed. In: Proceedings of The First Joint PSU-UNS International Conference on BioScience: Food, Agriculture, and the Environment, Thailand: 198-202.

11. Baskin, C.C. \& Baskin, J.M. (1998): Seeds: Ecology, biogeography and evaluation of dormancy and germination. San Diego, CA: Academic Press.

12. Forcella, F. (1998). Real-time assessment of seed dormancy and seedling growth for weed management. Seed Science Research, 8(2), 201-209. doi:10.1017/S0960258500004116

13. Forcella, F., Benech-Arnold, R.L., Sanchez, R. \& Ghersa, C.M. (2000). Modeling seedling emergence. Field Crops Research, 67, 123-139. 